\title{
New results on a Cn2 profiler for GeMS
}

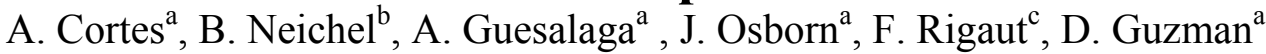 \\ ${ }^{\mathrm{a} P o n t i f i c i a ~ U n i v e r s i d a d ~ C a t o l i c a ~ d e ~ C h i l e, ~ V i c u n a ~ M a c k e n n a ~ 4860, ~ S a n t i a g o, ~ C h i l e ; ~}{ }^{\mathrm{b}} \mathrm{Gemini}$ \\ Observatory, Colina El Pino S/N, La Serena, Chile; ${ }^{\mathrm{C}}$ Australian National University Research School \\ of Astronomy and Astrophysics, Mount Stromlo Observatory, Cotter Road, Weston ACT 2611, \\ Australia
}

\begin{abstract}
The atmospheric optical turbulence profile, the strength of the turbulence as a function of altitude above the ground, can be used to determine the seeing statistics of a particular site. This information is useful for optimizing the tomographic process in Adaptive Optics systems and for characterizing the performance. In this paper, we describe a method to estimate the atmospheric turbulence profile based on the telemetry data coming out of GeMS, a Multi Conjugated Adaptive Optics (MCAO) instrument installed on the Gemini South telescope. The method is based on the SLODAR technique (SLOpe Detection and Ranging), where the wavefront slopes from two stars angularly separated on the sky are measured, and their cross-correlation is used to retrieve the atmospheric optical profile. We have modified the classical SLODAR method and adapted it for the closed loop, multiple laser guide stars case. In this paper we present our method, validation of it in simulation, and its application for on-sky data.
\end{abstract}

Keywords: $\mathrm{C}_{\mathrm{n}}^{2}$, Multi-Conjugate Adaptive Optics, Laser guide stars, Shack-Hartmann wavefront sensors, turbulence.

\section{INTRODUCTION}

$\mathrm{C}^{2}{ }_{\mathrm{n}}$ is the refractive index structure parameter that quantifies the strength of the atmospheric optical turbulence [1]. This parameter is widely used to characterize astronomical sites and for instance, determine the best location for the next generation of extremely large telescopes (ELT). It is also of prime importance for the tomographic process for Adaptive Optics wide field imaging. Usually, the $\mathrm{C}_{\mathrm{n}}^{2}$ profile is measured by dedicated instruments located outside of the telescope dome, or even at a different altitude than the telescope itself. Moreover, these instruments are also generally limited by their small collecting power, and they usually require bright stars. As a consequence, the stars used by these instruments (and hence direction in the sky) are different than the one observed by the astronomers [2]. This can result in bias in the $\mathrm{C}_{\mathrm{n}}{ }^{2}$ values between what is coming out of a dedicated instrument, and what the $\mathrm{AO}$ system needs. To solve this issue, we propose to use the telemetry from a multi-guide star $\mathrm{AO}$ system to recover the $\mathrm{Cn} 2$ profile, and feed the tomographic reconstructor.

For a system comprising several Shack-Hartmann Wave-Front Sensors (WFSs) such as the Gemini MCAO System (GeMS) installed at the Gemini South Observatory, SLOpe Detection And Ranging (SLODAR) [3][4][5] is a method that can be used to measure the turbulent profile. In its basic form, SLODAR estimates the relative strengths of turbulent layers at different altitudes by cross-correlating the information from two stars measured at a single WFS. The height resolution and range depend on the number of subapertures in the WFS and the angular separation between the stars. In a multi-guide stars system the conventional SLODAR technique is modified by using the data from multiple and independent WFSs. An example of the latter can be found in Wang et al [6] for a system using six possible baselines from four WFSs illuminated by natural guide stars.

We take this approach one step further by applying the technique to a system with Laser Guide Stars (LGS) that works in closed-loop. Therefore, aspects such as the cone effect, fratricide effect and pseudo-open loop slopes must be considered [7][8][9].

This paper is organized as follows. The first section describes the main characteristics of GeMS. The second section explains how the classical SLODAR method has been adapted in the case of GeMS. The third section shows how absolute measurement of the atmospheric parameters can be derived, and the next section illustrates the results with onsky data. Finally, section 6 assesses the limitations of the current method and the conclusions.

Adaptive Optics Systems III, edited by Brent L. Ellerbroek, Enrico Marchetti, Jean-Pierre Véran, Proc. of SPIE Vol. 8447, $84475 \mathrm{~T} \cdot$ ㅇ 2012 SPIE · CCC code: 0277-786/12/\$18 · doi: 10.1117/12.925242 


\section{GEMINI MCAO SYSTEM: GEMS}

The Gemini MCAO System (GeMS) is a multi-conjugate adaptive optics (MCAO) facility instrument installed at the Gemini South Observatory [10]. There are five laser guide star (LGSs) launched from behind the secondary mirror, and five wavefront sensors (WFSs), each pointing to one of the lasers. GeMS has three deformable mirrors (DMs) optically conjugated to the altitude of $0,4.5$ and $9 \mathrm{~km}$, work in closed loop to correct the effect of the atmospheric turbulence across the field of view of the telescope. GeMS is a unique facility installed on an 8-meter class telescope, providing a wide corrected field of 60 arc-seconds. More details on the system are shown on figure 1 and can be found in the paper Neichel et al [10].

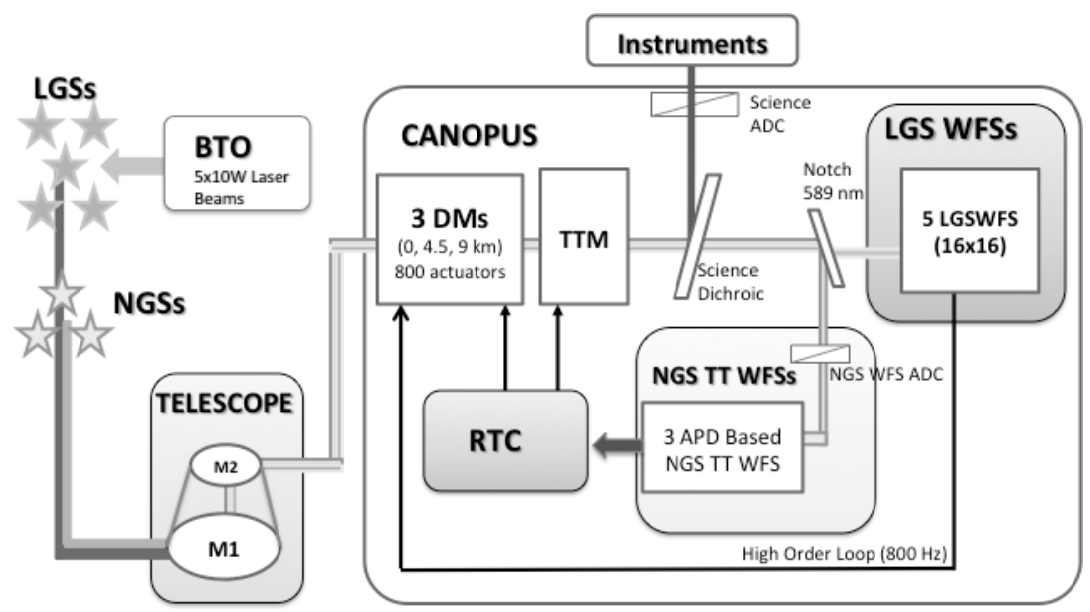

Figure 1. Simplified diagram of GeMS and CANOPUS.

The AO bench is mounted on the Cassegrain focus of the Telescope. The beam from the telescope is collimated by an off-axis parabola onto three piezo stack deformable mirrors (DMs) mentioned before, and a tip/tilt mirror (TTM). The main parameters of the DMs are summarized in table 1.

A science beam splitter feeds the instruments and reflects the light from the natural guide stars and the laser asterism. A notch filter reflects the five LGS beams and sends them to the five WFS that are aligned with the asterism. Each WFS is a 16x16 Shack-Hartmann, with 204 valid sub-apertures (shown in figure 2) sampled by $2 \times 2$ pixels (quadcell detector) with 3.5 e of measured read out noise. The maximum sampled frequency corresponds to $800 \mathrm{~Hz}$ for all the system.

Table 1. Main characteristics of the deformable mirrors.

\begin{tabular}{|l|c|c|c|}
\hline \multicolumn{1}{|c|}{ Item } & DM0 & DM4.5 & DM9 \\
\hline Pitch [mm] & 5 & 5 & 10 \\
\hline Active actuators & 240 & 324 & 120 \\
\hline Slave actuators & 53 & 92 & 88 \\
\hline Total & 293 & 412 & 208 \\
\hline
\end{tabular}




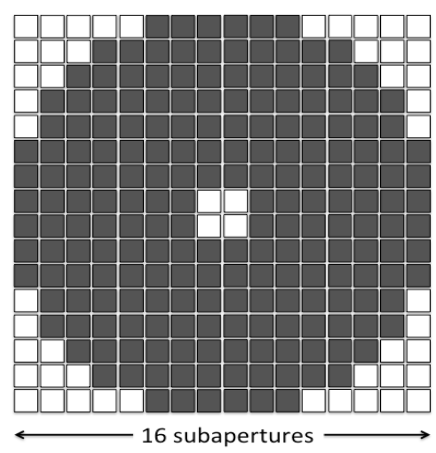

Figure 2. Active subapertures in dark, for each of the WFSs in GeMS

The laser asterism is formed in the Beam Transfer Optics (BTO), and it consists of a 1 arcmin-side square with a fifth LGS at the center of it. Each LGS has a power of $10 \mathrm{~W}$ and the stars are created at an altitude of approximately $90 \mathrm{~km}$ when propagating at Zenith.

The five beams are launched from behind the secondary mirror of the telescope.

\section{METHOD TO MEASURE THE TURBULENCE PROFILE}

Several methods and instruments exist to measure the characteristics of the optical turbulence of the atmosphere. In our case, the most convenient one was to start from a SLODAR approach. This method uses the spatial cross correlation of the local gradient of the wavefront phase aberration measured at the ground level using SH-WFS [4][5][11]. As GeMS already complies with several WFSs and several guide sources, the SLODAR implementation was straightforward. Three main modifications to the classical SLODAR approach are however required.

First the SLODAR is usually implemented with natural stars while the WFS of GeMS observe LGS at finite distance. This means that we have to consider the so-called cone effect from the measurement. Second, the SLODAR method operates with two stars, while GeMS uses five stars, bringing us more baselines and sampling capabilities. Third, the SLODAR method works in open-loop, while the telemetry data from GeMS is closed loop. In addition, there are a few more differences exist in the data handling and pre-calibration which are discussed further in section 5.

To explain how the technique estimates the turbulence profile it is important to see the layer structure for the optical triangulation. A simplified example for two LGS is represented in figure 3, laser guide stars are formed at a finite altitude, hence as described above the area of the sampled atmosphere decreases for higher altitudes. This compression effect modifies the altitude at which the SLODAR reconstructs the turbulence [7][8][9]. For a WFS with $N$ subapertures across the pupil of the telescope (ground level), the telescope diameter can be divided into $\mathrm{N}$ areas of size $d$, that determinate the number of layers that can be measured. The central altitude of each layer is given by $h=m d /(\theta \sec \zeta)$, where $m=\{1, . ., \mathrm{N}-1\}$ is an integer that identifies the layer or bin. $\theta$ is the relative angular separation between the stars and $\zeta$ is the zenith angle. The altitude of these discrete layers, when the telescope is pointing at zenith, is given by:

$$
h_{m}=\frac{m d z}{z \theta+m d}
$$

where $z$ is the altitude of the sodium layer. If we subtract two levels of $h_{m}$, we can get the approximated equation for:

$$
\delta h_{m}=\frac{d z^{2} \theta}{(z \theta+m d)^{2}}
$$




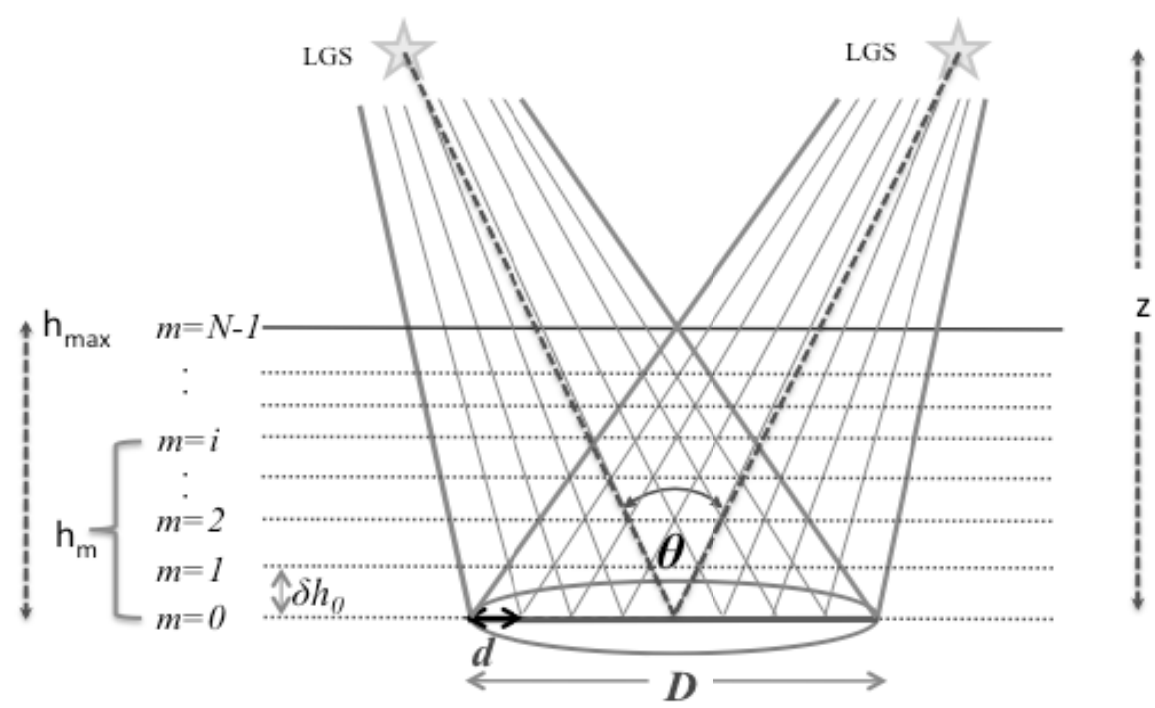

Figure 3.Diagram of two laser stars separated on sky by an angle $\theta$, observed by WFS with NxN sub-apertures. D is the telescope pupil diameter.

In the SLODAR method, the optical triangulation is between two stars and depends on the separation angle on the sky $\theta$. Considering the asterism topology of GeMS, we can get up to 10 baselines to measure, and the altitude resolution depends on the angular separation. Using the GeMS structure, we have three different angular separation (see figure 4) giving multiple altitude resolutions. The explanation of the resolutions is given by using the equation (2), and the square configuration of 60 arcseconds on the side, with one star in the middle.

i) Horizontal and vertical baselines between stars at the corner (4 baselines, figure 4 left)

$$
\delta h_{m}=\frac{d z^{2} \theta}{(z \theta+m d)^{2}}=\frac{0.5 \cdot 60^{\prime \prime} z^{2}}{\left(60^{\prime \prime} z+0.5 m\right)^{2}}
$$

ii) Diagonal baselines between stars at the corners ( 2 baselines, figure 4 central image)

$$
\delta h_{m}=\frac{\sqrt{2} d z^{2} \theta}{(z \theta+m \sqrt{2} d)^{2}}=\frac{0.5 \cdot \sqrt{2} \cdot 60^{\prime \prime} \cdot \sqrt{2} z^{2}}{\left(60^{\prime \prime} \cdot \sqrt{2} \cdot z+0.5 \cdot \sqrt{2} \cdot m\right)^{2}}=\frac{0.5 \cdot 60^{\prime \prime} \cdot z^{2}}{\left(60^{\prime \prime} \cdot z+0.5 \cdot m\right)^{2}}
$$

iii) Diagonal baselines between the central stars and the ones at the corners ( 4 baselines, figure 4 right)

$$
\delta h_{m}=\frac{\sqrt{2} d z^{2} \theta}{(z \theta+m \sqrt{2} d)^{2}}=\frac{0.5 \cdot \sqrt{2} \cdot 30^{\prime \prime} \cdot \sqrt{2} \cdot z^{2}}{(30 " \cdot \sqrt{2} \cdot z+0.5 \cdot \sqrt{2} \cdot m)^{2}}=\frac{0.5 \cdot 30 " \cdot z^{2}}{(30 " \cdot z+0.5 \cdot m)^{2}}
$$

The maximum altitude to sample is given by the low resolution case, i.e. the angular separation of 42.4 arcsec and an altitude of $32.78 \mathrm{~km}$. The high resolution will give a better sampling but a lower maximum altitude, this is given by the angular separation of 84.9 arcsec and 60 arcsec. 


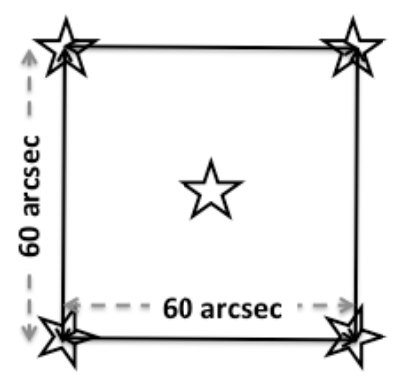

i)

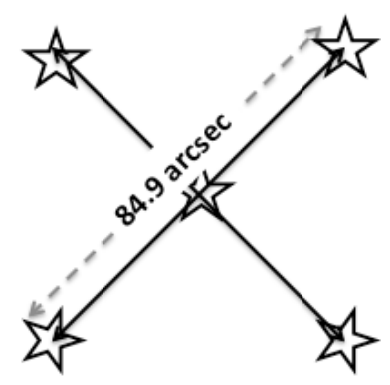

ii)

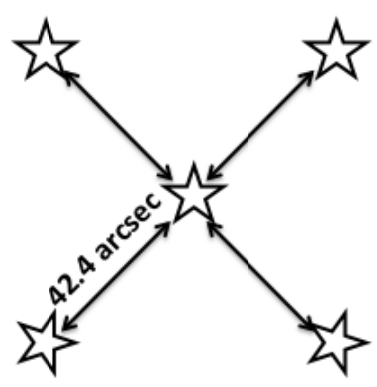

iii)

Figure 4. Three cases of angular separation for the LGS on GeMS. The high resolution (low $\left.h_{\max }\right)$ is given by i) and ii). The low resolution (and higher $\mathrm{h}_{\max }$ ) is given by iii).

\section{GeMS data structure}

The main difference between SLODAR and GeMS is that the slopes used with the SLODAR technique are integrated through the complete turbulent volume, while GeMS is measuring only the residual of the slopes after the correction by the AO system. It was thus necessary to reconstruct the complete slopes of the incoming wavefront, which we call pseudo open loop (POL). This procedure uses the information of the voltages applied to the DM and the interaction matrix $i$ Mat (the static response of an AO system), using the following equation:

$$
S_{i}^{p o l}=S_{i}^{r e s}+i M a t^{*} V_{i-1}^{a c t}
$$

where $i$ is the discrete time, $S_{i}^{r e s}$ correspond to the residual slopes that are measured by GeMS. The size of the $i M a t$ is $N_{m s} \times N a_{c t}$, where $N_{a c t}$ is the number of actuators of the system (684 actuators here).
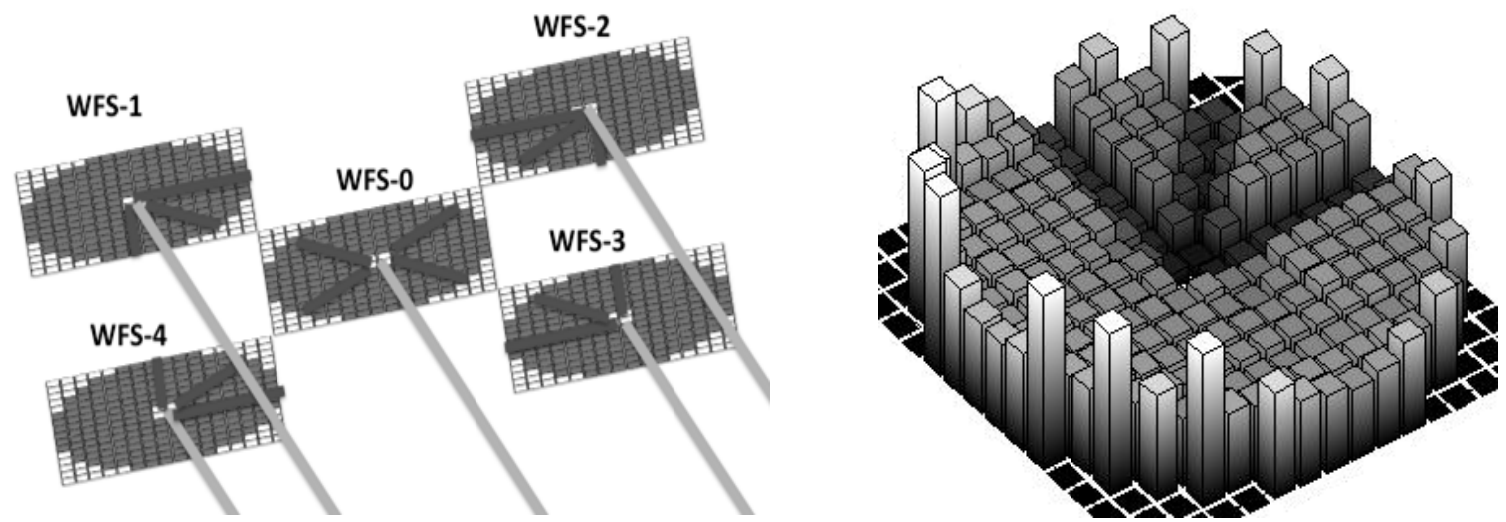

Figure 5. The fratricide effect is when one of the WFS sees the scattered light produced by the adjacent LGS. The figure on the left is the explicative diagram, and the one to the right the real RMS for the slopes of one WFS.

As the LGSs in GeMS are launched from behind the secondary mirror, the Rayleigh scattering from the adjacent LGSs are seen in the WFS, producing a light contamination known as the fratricide effect (see figure 5). The right image corresponds to the RMS of the centroid data taken on sky. Sub-apertures affected by the fratricide have a much lower variance than properly illuminated ones. In addition to the fratricide, subapertures at the edge of the pupil show a higher variance, due to the fact that they are only partially illuminated. 
It was necessary to create a mask to eliminate the fratricide effect and the vignetted subapertures. The method to filter them out automatically has been described in Cortes AO4ELT2 [8]. This decreases the number of available subapertures to correlate in the SLODAR method, which also means a smaller maximum altitude that can be sampled (see table 2).

Table 2. Number of subapertures available for correlation, at different altitudes. The grey cells indicate the values used for the analysis.

\begin{tabular}{|c|c|c|c|c|}
\cline { 2 - 5 } \multicolumn{1}{c|}{} & \multicolumn{2}{c|}{ High resolution } & \multicolumn{2}{c|}{ Low resolution } \\
\hline Bins & $\begin{array}{c}\text { Altitude } \\
{[\mathrm{Km}]}\end{array}$ & $\begin{array}{c}\text { Overlapping } \\
\text { subapertures } \\
{[\#]}\end{array}$ & $\begin{array}{c}\text { Altitude } \\
{[\mathrm{Km}]}\end{array}$ & $\begin{array}{c}\text { Overlapping } \\
\text { subapertures } \\
{[\#]}\end{array}$ \\
\hline 15 & 20.0 & - & 32.8 & - \\
\hline 14 & 19.0 & - & 31.4 & - \\
\hline 13 & 17.9 & 24 & 29.9 & - \\
\hline 12 & 16.8 & 48 & 28.3 & - \\
\hline 11 & 15.6 & 68 & 26.6 & - \\
\hline 10 & 14.4 & 84 & 24.9 & 8 \\
\hline 9 & 13.2 & 108 & 23.0 & 32 \\
\hline 8 & 11.9 & 144 & 21.1 & 64 \\
\hline 7 & 10.6 & 164 & 19.0 & 72 \\
\hline 6 & 9.3 & 172 & 16.8 & 72 \\
\hline 5 & 7.8 & 252 & 14.4 & 96 \\
\hline 4 & 6.4 & 328 & 11.9 & 136 \\
\hline 3 & 4.9 & 396 & 9.3 & 216 \\
\hline 2 & 3.3 & 460 & 6.4 & 272 \\
\hline 1 & 1.7 & 536 & 3.3 & 320 \\
\hline 0 & 0 & 624 & 0 & 368 \\
\hline & & & & \\
\hline
\end{tabular}

\section{Simulations}

To validate the modified SLODAR approach in the GeMS framework, we have conducted a full and comprehensive set of simulations.

The first step of a simulation corresponds to creating phase screens that represent real turbulence. The standard Kolmogorov model of the turbulence was used. It considers that the spatial spectrum of aberrations at the ground follows the equation [7]:

$$
\Phi(k)=0.023 r_{0}^{-5 / 3} k^{-11 / 3}
$$

The algorithm uses cross-covariance of the centroid slope measurements. The response functions of the system can be calculated theoretically [8], or in Monte-Carlo simulation. We used the Monte-Carlo simulation, assuming that the integrated turbulence can be represented as a sum of thin layers positioned at the altitude of each bin (table 2). Then the resulting phase at ground level for each WFS $\varphi_{j}^{\text {sim }}$ is transformed into slopes by simulating the WFSs of GeMS via the $D^{W F S}$ matrix:

$$
S_{j}^{s i m}=D^{W F S} \varphi_{j}^{s i m}
$$


where $j$ is the index of the bin.

The simulations are made for the subapertures after the masking, and follow the steps described in reference [12] with a phase-screen size of $4000 \times 4000$ pixels. An adequate length for the simulated sequence was found to be 10,000 frames. The theoretical covariance matrix for slopes in layer $m$ is defined as:

$$
\mathrm{C}_{m}^{s i m}=\frac{1}{10,000} \sum_{i=1}^{10,000} S_{m, i}^{s i m} \cdot\left(S_{m, i}^{s i m}\right)^{T}
$$

The set of resulting matrices contains the correlation between the slopes, with a size of $2040 \times 2040$. To reduce the size, we use covariance maps $\mathrm{M}_{j}^{\text {sim }}$, where the data are grouped and rearranged using the advantage of the spatial redundancies between subapertures, generating matrices with a size of 330 x 330 for each bin (see figure 6 left).

The diagonal of each covariance map corresponds to autocorrelations for each of the 5 WFS, and the off-diagonals contain the cross-correlations. Figure 6 shows the covariance map $\mathrm{M}_{j}^{\text {sim }}$ for bin 6 . The slopes are split into $\mathrm{X}$ and $\mathrm{Y}$, and then into each WFS. This matrix contains $10 \times 10$ sub-maps, and as one can see, the peaks in the cross-correlations are displaced from the center (see figure 6, bottom right), according to their relative position. This means that for the bin 6 , by example, it will be displaced by 6 pixels in the direction of the two WFSs.

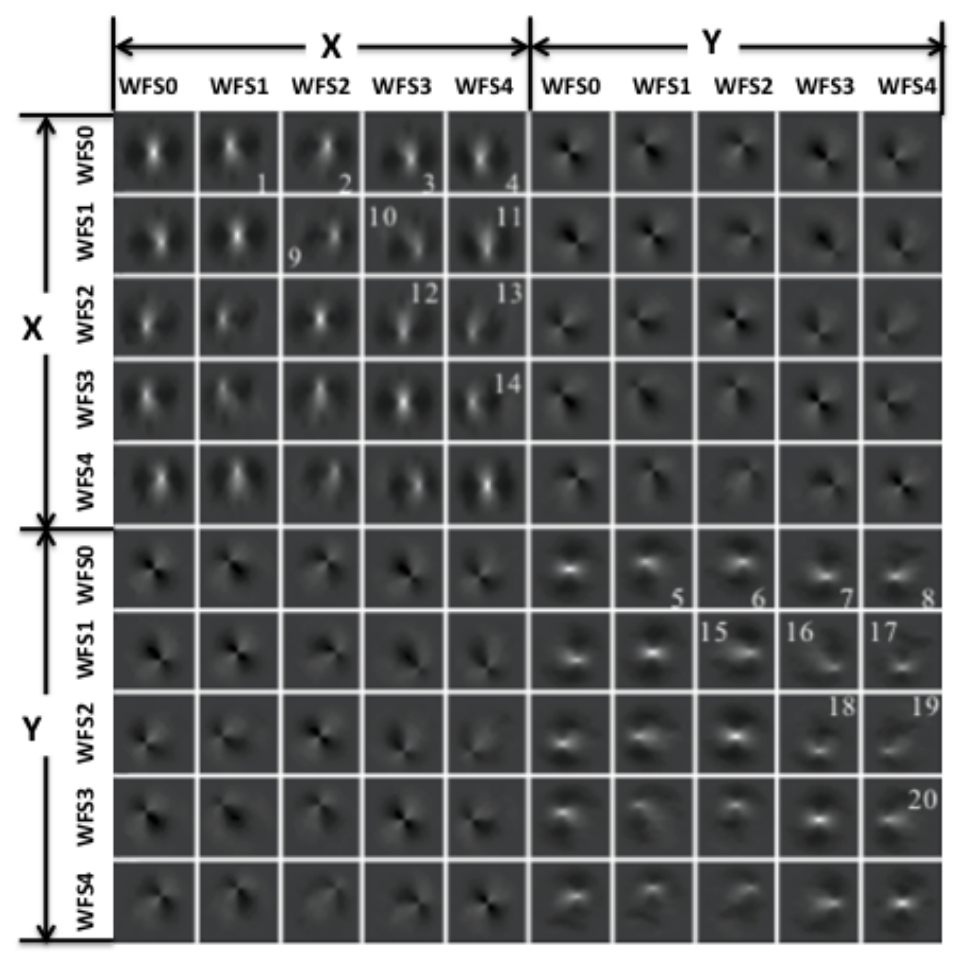

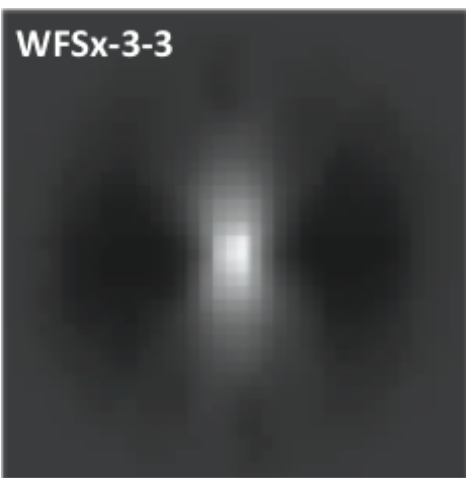

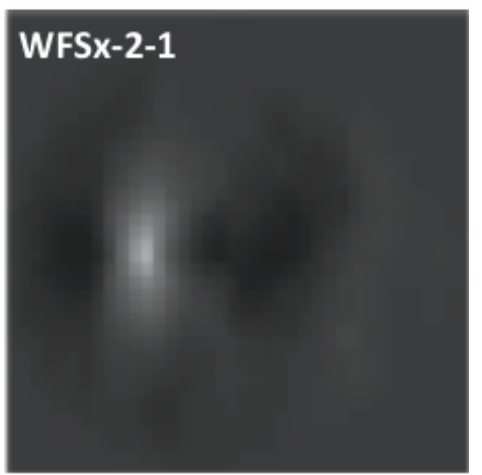

Figure 6. Left, theoretical covariance map for bin $6, \mathrm{M}_{6}^{\text {sim }}$. Bin 6 corresponds to $9.3 \mathrm{~km}$ for the high resolution, and $16.8 \mathrm{~km}$ for the low resolution. Right, a zoom of two sub-maps.

For the covariance maps, we also have some symmetry, as it can be seen in figure 6 . There are only 20 non-redundant sub-maps (numbered in white in the figure 6) that we use. The cross-correlations between X and Y are not used. The first 8 sub-maps correspond to the cross-correlations between the WFS in the middle (WFS0) with the WFSs at the corners, represented by $\mathbf{M}_{j, L R}^{\text {sim }}=\left\{\mathbf{M}_{j, 1}^{\text {sim }}, . ., M_{j, 8}^{\text {sim }}\right\}$, i.e. the low resolution. The high resolution is determined by the sub-maps from 9 to 20, between the corners (sides and diagonal), and is represented by $\mathbf{M}_{j, H R}^{\operatorname{sim}}=\left\{\mathbf{M}_{j, 9}^{\mathrm{sim}}, \ldots, \mathbf{M}_{j, 20}^{\mathrm{sim}}\right\}$. 


\section{Fitting}

For the data measured on sky, we have only one covariance map, that we will call $\mathrm{M}_{j}^{\text {meas }}$, where $j$ will indicate the submap index. To determine the profile of the turbulence in layers, we need to estimate the weights that are expressed by vectors that fulfill the equations 9 and 10 .

$$
\begin{array}{cl}
\underset{\omega^{L R}}{\operatorname{Min}} & \sum_{j=1}^{8} W_{j} *\left\lfloor\left(\sum_{m=0}^{L_{L R}-1} \omega_{m}^{L R} \mathrm{M}_{m, j}^{\text {sim }}\right)-\mathrm{M}_{j}^{\text {meas }}\right\rfloor \\
\underset{\omega^{H R}}{\operatorname{Min}} & \sum_{j=9}^{20} W_{j} *\left\lfloor\left(\sum_{m=0}^{L_{H R}-1} \omega_{m}^{H R} \mathrm{M}_{m, j}^{\text {sim }}\right)-\mathbf{M}_{j}^{\text {meas }}\right\rfloor
\end{array}
$$

This means that using the simulated covariance maps, we determine the weights that will give us the best match with the measured data. In the equations above, $W_{j}$ corresponds to a mask for sub-map $j$ that selects only those values of $\mathbf{M}_{m, j}^{\text {sim }}$

and $\mathrm{M}_{j}^{\text {meas }}$ with high signal to noise ratio (from the center to the edge in the direction between the two WFSs). Vectors $\omega_{m}^{L R}$ and $\omega_{m}^{H R}$ contain the coefficients that weight the theoretical maps for the LR and HR cases respectively. It must be noted that the operator ' $*$ ' is a matrix point-to-point multiplication.

For this minimization, we used the Truncated Least Square technique [13], because it always converges to a global minimum and is faster than other methods.

\section{ABSOLUTE VALUES FOR $\mathbf{C}_{\mathbf{n}}^{2}$}

The previous method gives us the relative profile of the turbulence. Absolute values for the turbulence strength can then be estimated by using the variance and the equation that relates the Fried Parameter $\left(r_{0}\right)$ with $C_{n}^{2}[14]$ :

$$
r_{0}=\left[0.423 k^{2} \sec \varsigma \int_{0}^{H} C_{n}^{2}(z) d z\right]^{-3 / 5}
$$

The integral of the profile will correspond, in our assumptions, to:

$$
\int_{0}^{H} C_{n}^{2}(z) d z=\sum_{m=0}^{N-1} C_{n}^{2}(m) \cdot \delta h_{m}
$$

considering $H$ as the maximum altitude used to calculate the profile, this depends on the resolution used.

Assuming that the Fried Parameter can be estimated in layers $r_{0}(m)$, and assuming that the total Fried parameter will be the sum of all of them, we can obtain:

$$
C_{n}^{2}(m) \delta h_{m}=\frac{1}{0.423 k^{2} \sec \varsigma} \cdot \frac{r_{0}(m)^{-5 / 3}}{\rho_{m}}
$$

and $\rho_{m}$ is the stretching factor for $r_{0}$, considering the impact of the cone effect. This optical spatial expansion is given by:

$$
\rho_{m}=1-h_{m} / z
$$

Considering the tilt variance integrated over a subaperture, with diameter $d$, we know from [15]: 


$$
\sigma_{d}^{2}=0.179 \lambda^{2} r_{0}^{-5 / 3} d^{-1 / 3}
$$

Defining $\sigma_{0}^{2}$ as the sum of the subapertures variances for the theoretical slopes (just the valid ones), and using the results of the equations (9) and (10), we can express the turbulence strength for the layer $m$ as:

$$
C_{n}^{2}(m) \cdot \delta h_{m}=\frac{2.37 \cdot \omega_{m}}{\sec \zeta} \sigma_{0}^{2}
$$

To determine the full turbulence, it is important to add the turbulence above the highest bin, which requires the use of information from the autocorrelations. We call the sub-maps of the diagonal in figure $6 \mathrm{~V}^{\text {meas }}=\left\{V_{1}^{\text {meas }}, . ., V_{p}^{\text {meas }}, \ldots, V_{10}^{\text {meas }}\right\}$, where $\mathrm{p}$ indicates the position along the diagonal.

The central point corresponds to the slope variance plus the noise variance, but the theoretical impulse functions $V^{\text {sim }}$ have no noise. Therefore subtracting these two values will give us an estimate for the noise variance. This can be estimated by:

$$
\underset{\eta}{\operatorname{Min}} \sum_{p=1}^{10} U_{p} *\left(\eta V_{p}^{\text {sim }}-V_{p}^{\text {meas }}\right)
$$

where $p$ and $U_{p}$ is a mask that eliminates the central point of the submaps [5].

We can now eliminate the noise from the measured autocovariance sub-maps, and get the total turbulence above the telescope, determined as the slope variance in the 5 WFS. Then, from equations (16) and (17), we get the integrated turbulence strength:

$$
\int_{0}^{\infty} C_{n}^{2}(h) \cdot d h=\frac{2.37}{\sec \zeta} \cdot \eta \cdot \sigma_{0}^{2}
$$

\section{RESULTS ON-SKY}

Using the method to obtain the $\mathrm{C}_{\mathrm{n}}^{2}$ profile as described in section 3, we determine the strength of the turbulence in bins up to the maximum altitude presented in table 2, and using the process explained in section 4 the information was completed to obtain the full profile. In figure 7, we present six different profiles, taken on different nights. The figures (c), (d), (e) and (f) are from the same night at different times. The calculated Fried parameter was around $r_{0}=15 \mathrm{~cm}$ in these profiles, for the analyzed night, the $\mathrm{r}_{0}$ was between $23 \mathrm{~cm}$ and $6.8 \mathrm{~cm}$. It can be appreciated that the strongest turbulences is located in the first bin, and the "unsensed" turbulence that correspond to the turbulence above the maximum limit that can be measured with the SLODAR method. The "unsensed" turbulence is calculated using the variance (explained on the previous section). 


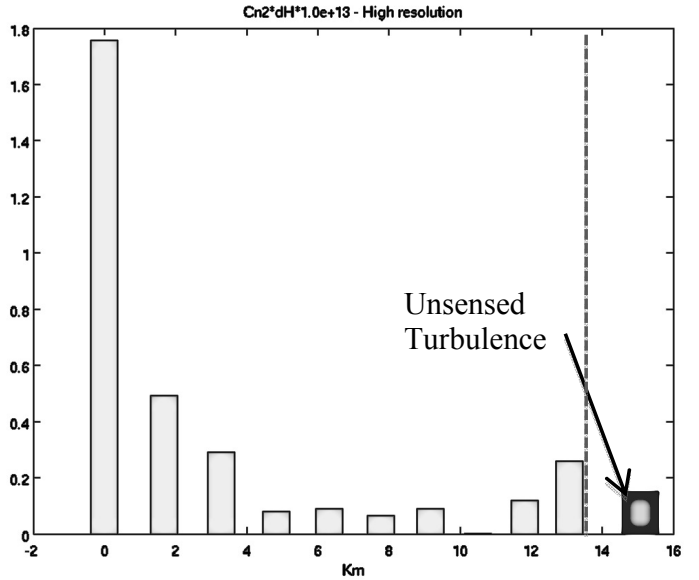

(a)

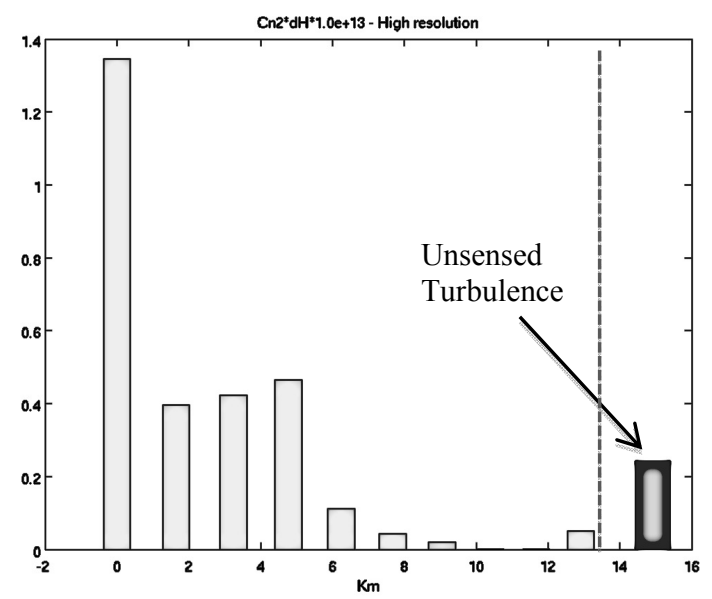

(c)

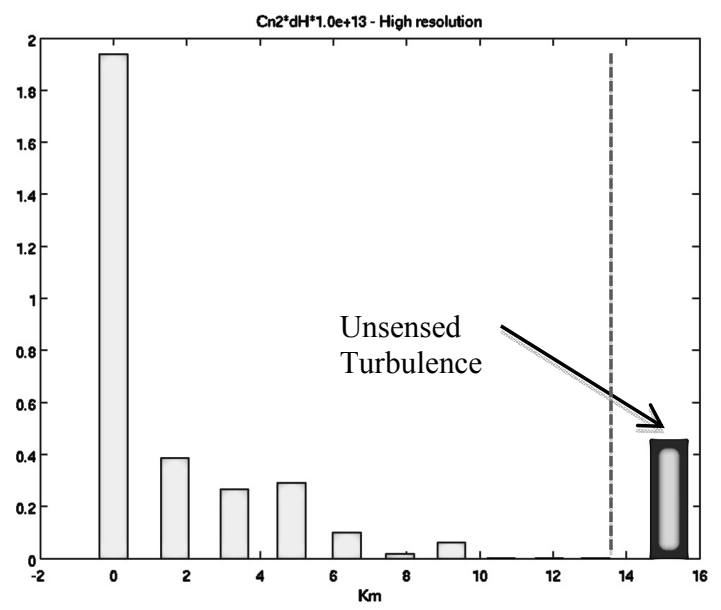

(e)

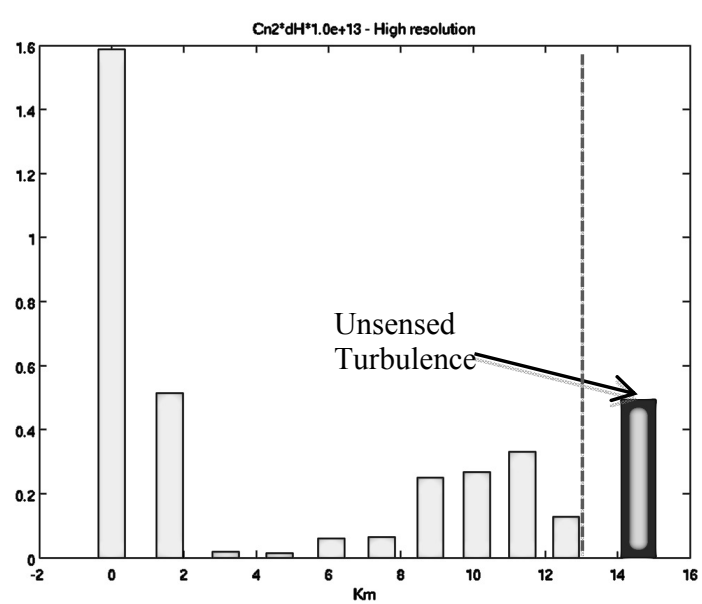

(b)

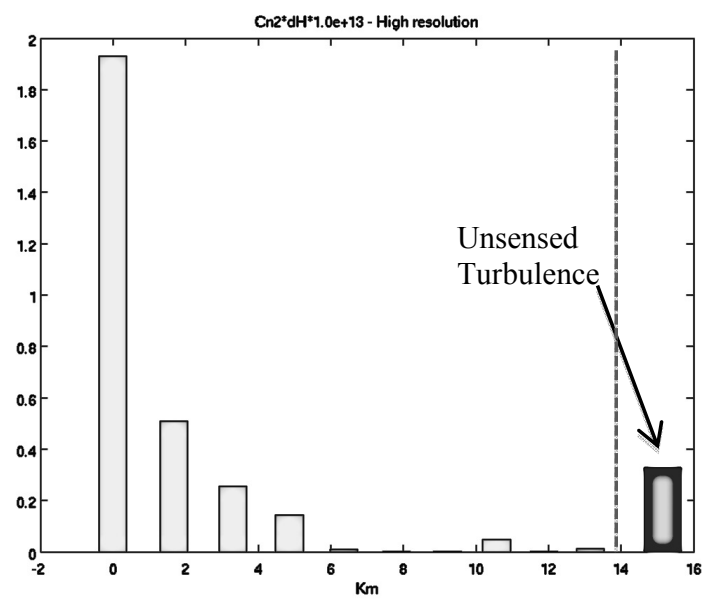

(d)

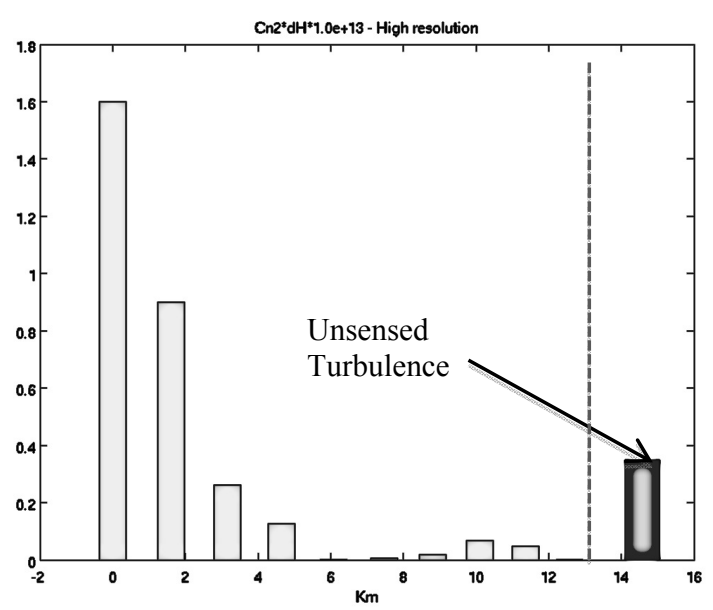

(f)

Figure 7. Six different profiles $\left(\mathrm{C}_{\mathrm{n}}{ }^{2}\right)$, results of the fitting process when applying the SLODAR method, with the LGSs. The dashed line shows the maximum altitude that can be measured, in this case, for the high-resolution. The Fried parameter is calculated with the profile. (a) correspond to the night 10/15/12 at 21:36:51, (b) correspond to the night 13/03/12 at 01:13:01, (c) correspond to the night 16/03/12 at 00:19:03, (d) correspond to the nigh 16/03/12 at 00:57:33, (e) correspond to the nigh 16/03/12 at 01:03:38 and (f) correspond to the nigh 16/03/12 at 03:12:32. 


\section{CONCLUSIONS}

A detailed analysis of the possible sources of error associated with our method is presented in [9]. We summarize here the main findings. First, one possible source of errors lies in the POL reconstruction. This reconstruction relies on the assumption that the centroid gains of the LGSWFS are perfectly calibrated. An error in these centroid gains would impact the reconstructed profiles. To quantify this effect, we ran simulations by introducing random noise to the measured slopes, and we studied how those errors would propagate on the $\mathrm{C}_{\mathrm{n}}{ }^{2}$ profile reconstruction. We found that on the high-resolution case, the errors fell below $3 \%$ for deviations in the centroid gain of up to $50 \%$ respect to the correct value. For the low-resolution case, however, these errors jumped to $8 \%$ for a $50 \%$ deviation.

It is interesting to note that this error had little impact for relative profiles (normalized contributions of each layer to the total turbulence strength), for both high and low resolution. Furthermore, GeMS can calibrate the centroid gains in almost real time during on-sky operation [16], so the negative impact can be known and limited. On the other hand, we also run for different values of the turbulence outer scale $\mathrm{L}_{0}$, but no significant impact was found on the result. This is not surprising, since by eliminating the tip and tilt from the POL slopes, the potential effect of differences in the theoretical and measured the lower part of the spectrum is greatly attenuated.

More characterization of the method using calibrated turbulence introduced by the DM has been performed, and in all cases our method had proven to be robust and providing accurate results. More details are given in [9]. This gives us a good confidence level in the results presented above, and demonstrates that estimating the turbulence profile based on the telemetry data out of a multi-LGS instrument is possible.

More data is currently under analysis. This data will be used to characterize GeMS performance obtained during its first months of operations. We are also building a data base, and deriving statistics, that could be eventually used for optimizing the scheduling of GeMS. Finally, the $\mathrm{C}_{\mathrm{n}}{ }^{2}$ profiler will soon be integrated into the GeMS real time software, and be used for on-line optimization of the tomographic matrices.

\section{ACKNOWLEDGMENTS}

This work has been supported by the Chilean Research Council (CONICYT) through grant Fondecyt 1120626, Anillo ACT-86 and scholarship for first author. We are also grateful to Andrei Tokovinin, Tim Butterley and Tim Morris for fruitful discussions. Part of this work has been supported by Gemini. The Gemini Observatory is operated by the Association of Universities for Research in Astronomy, Inc., under a cooperative agreement with the NSF on behalf of the Gemini partnership: the National Science Foundation (United States), the Science and Technology Facilities Council (United Kingdom), the National Research Council (Canada), CONICYT (Chile), the Australian Research Council (Australia), Ministério da Ciência e Tecnologia (Brazil) and Ministerio de Ciencia, Tecnología e Innovación Productiva (Argentina).

\section{REFERENCES}

[1] A. Glindemann, S. Hippler, T. Berkefeld, and W. Hackenberg, "Adaptive optics on large telescopes," Experimental Astronomy 10, 5-47 (2000).

[2] J. Thomas-Osip, E. Bustos, and M. Goodwin, "Two campaigns to compare three turbulence profiling techniques at Las Campanas Observatory," Proc. SPIE 7014, 196 (2008).

[3] R. Wilson, "SLODAR: measuring optical turbulence altitude with a Shack-Hartmann wavefront sensor," Monthly Notices of the Royal Astronomical Society 337, 103-108 (2002).

[4] R. W. Wilson, T. Butterley, and M. Sarazin, "The Durham/ESO SLODAR optical turbulence profiler," Monthly Notices of the Royal Astronomical Society 399, 2129-2138 (2009).

[5] T. Butterley, R. W. Wilson, and M. Sarazin, "Determination of the profile of atmospheric optical turbulence strength from SLODAR data," Monthly Notices of the Royal Astronomical Society 369, 835-845 (2006).

[6] L. Wang, M. Schöck, and G. Chanan, "Atmospheric turbulence profiling with SLODAR using multiple adaptive optics wavefront sensors.," Applied optics 47, 1880-1892 (2008). 
[7] L. Gilles and B. Ellerbroek, "Real-time turbulence profiling with a pair of laser guide star Shack-Hartmann wavefront sensors for wide-field adaptive optics systems on large to extremely large telescopes," JOSA A 27, A76A83, (2010).

[8] A. Cortés, B. Neichel, A. Guesalaga1, et al, "First results on a Cn2 profiler for GeMS" presented at the second AO4ELT Conference (Victoria, Canada), (2011).

[9] A. Cortés, B. Neichel, A. Guesalaga, and et al., "Atmospheric turbulence profiling using multiple laser star wavefront sensors", MNRAS, submitted (2012).

[10] B. Neichel, F. Rigaut, M. Bec, M. Boccas, F. Daruich, C. D’Orgeville, V. Fesquet, R. Galvez, A. Garcia-Rissmann, et al., "The Gemini MCAO System GeMS: nearing the end of a lab-story," in Proceedings of SPIE 7736, p. 773606 (2010).

[11]R. Wilson, "SLODAR: measuring optical turbulence altitude with a Shack-Hartmann wavefront sensor", Monthly Notices of the Royal Astronomical Society 337, 103-108 (2002).

[12] McGlamery B.L., "Computer simulation studies of compensation of turbulence degraded images", Image Processing, SPIE 74, 225-233 (1976).

[13] G. H. Golub and C. F. Van Loan, "An analysis of the total least squares problem", SIAM Journal on Numerical Analysis, Vol. 17, No. 6, pp. 883-893 (1980).

[14] J. W. Hardy, [Adaptive Optics for Astronomical Telescopes], Oxford University Press, New York, (1998).

[15]D. L. Fried, "Differential angle of arrival: theory, evaluation, and measurement feasibility", Radio Science, 71-76 (1975).

[16]F. Rigaut, B. Neichel et al., "Gemini south MCAO on-sky results", presented at the second AO4ELT Conference (Victoria, Canada), (2011). 